\title{
Baseline ventilatory function predicts the development of higher levels of fasting insulin and fasting insulin resistance index: the Normative Aging Study
}

\author{
R. Lazarus*+, D. Sparrow ${ }^{\ddagger}$, S.T. Weiss ${ }^{+}$
}

\begin{abstract}
Baseline ventilatory function predicts the development of higher levels of fasting insulin and fasting insulin resistance index: the Normative Aging Study. R. Lazarus, D. Sparrow, S.T. Weiss. CERS Journals Ltd 1998.

ABSTRACT: A consistent but as yet unexplained association between baseline ventilatory function and risk of coronary heart disease (CHD) has been reported from many prospective studies. Insulin-resistant states are associated with increased risk of CHD.

Forced vital capacity (FVC), forced expiratory volume in one second (FEV1) and maximal mid-expiratory flow rate (MMEF) at study entry were examined as predictors for indirect measures of insulin resistance after a mean follow-up interval of $\mathbf{2 0 . 9}$ yrs in 1050 nondiabetic male subjects in the Normative Aging Study.

Males in the top quintile of insulin or fasting insulin resistance index (FIRI) levels at follow-up were defined as being relatively insulin resistant. FVC was negatively associated with risk of being relatively insulin resistant using the insulin $(p=0.002)$ or FIRI $(p=0.0001)$ criteria at follow-up in logistic regression models adjusting for baseline age, body mass index, fat distribution pattern and cigarette smoking. Similar associations were found for FEV1 and MMEF. Additional adjustment for baseline postcarbohydrate challenge glucose levels made little difference to the results, suggesting that baseline glucose intolerance was not a significant source of bias.

These findings are consistent with the possibility that insulin resistance may be one of the factors mediating the previously unexplained prospective association between impairment of ventilatory function and risk of mortality from coronary heart disease. Eur Respir J 1998; 12: 641-645.
\end{abstract}

*Dept of Public Health and Community Medicine, Faculty of Medicine, University of Sydney, Australia. +Channing Laboratory, Dept of Medicine, Brigham and Women's Hospital, Harvard Medical School, Boston, USA. *Normative Aging Study, Dept of Veterans Affairs, Boston, USA.

Correspondence: R. Lazarus, Faculty of Medicine, A27, University of Sydney, Camperdown NSW 2006, Australia. Fax: 61293515049

Keywords: Coronary disease, insulin resistance, prospective study, serum insulin levels, spirometry

Received: August 131997

Accepted after revision 1998

Supported by grants HL-37871 and HL 45089 from the National Heart Lung and Blood Institute, grant MO1RR01032 from the General Clinical Research Center, Beth Israel Hospital and the Health Services Research and Development Service of the Dept of Veterans Affairs. D. Sparrow is an Associate Career Scientist from the VA Medical Research Service. R. Lazarus was supported by a Special Studies Program from the University of Sydney.
The association between an increased risk of cardiovascular mortality and lower baseline levels of ventilatory function remains largely unexplained, even though it has long been recognized and consistently reported in prospective population studies [1-4]. Several mechanisms have been proposed [5] but there is little observational evidence to support any of these.

A number of cross-sectional studies have reported negative unadjusted correlations between ventilatory function and markers of glucose intolerance [6-8]. In a prospective study, the development of diabetes was associated with greater rates of decline in ventilatory function over a five year follow-up period [9]. Insulin resistance, variously defined [10], is associated with a range of cardiovascular risk factors including dyslipidaemia, essential hypertension, glu-cose intolerance and diabetes [11]. If lower levels of ventila-tory function precede the development of insulin resistance, the association of ventilatory function with cardiovascular mortality may be at least partially mediated by insulin res-istance.

The purpose of this report was to examine the relationship between ventilatory function at study entry and the development of insulin resistance in a relatively large cohort of males in the Normative Aging Study (NAS). It was hypothesized that lower levels of ventilatory function at baseline were associated with a greater risk of being relatively insulin resistant at follow-up.

\section{Methods}

The NAS is an ongoing longitudinal study established by the Veterans Administration in 1961 which has already been described in detail [12]. Volunteers were screened according to specific clinical, laboratory, spirometric, radiological, electrocardiographic and medical history criteria [12] and the cohort comprised 2,280 community-dwelling males from the Boston area, aged 21-80 yrs at entry. The study protocol was approved by the Human Studies Subcommittee of the Research and Development Committee, Department of Veterans Affairs Outpatient Clinic. Written informed consent was obtained from all subjects.

Participants are re-examined periodically and anthropometry is performed at each visit. Body mass index (BMI) [13] was calculated as the weight in kilograms divided by the square of the height in metres $\left(\mathrm{kg} \cdot \mathrm{m}^{-2}\right)$. Abdominal girth was measured at the level of the umbilicus, and hip breadth was measured at the level of the greater trochanters with an anthropometer. The ratio of abdominal circumference to hip breadth (AC/HB) was used as a measure of a central pattern of fat distribution. 
Spirometry was performed in the standing position, without a noseclip, using a water-filled recording spirometer (Warren E. Collins, Braintree, MA, USA). Each subject made three acceptable forced vital capacity (FVC) manoeuvres according to predefined criteria [14] and standard methods [14] were used to obtain the FVC (L), forced expiratory volume in one second $\left(\mathrm{FEV}_{1} ; \mathrm{L}\right)$ and maximal midexpiratory flow (MMEF; $\left.\mathrm{L} \cdot \mathrm{S}^{-1}\right)$. All values were corrected to body temperature and pressure saturated with water vapour (BTPS).

Subjects fasted overnight before their examination. At each visit, fasting blood samples were drawn and an oral glucose load of $100 \mathrm{~g}$ was given. Two hours later, blood was drawn for measurement of $2 \mathrm{~h}$ postchallenge glucose levels (PC glucose). For a limited period (from February 1987 to July 1991), insulin and glucose levels from fasting samples were measured. The insulin data from this limited period formed the basis of this report. Insulin was measured using a solid-phase 125I-radioimmunoassay (Coat-ACount Insulin 1987, Diagnostic Products Corporation, Los Angeles, CA, USA). The interassay and intra-assay coefficients of variation were $5-7 \%$ and $3-5 \%$, respectively. The fasting insulin resistance index (FIRI) [15] is an algebraically simplified form of the Homeostasis Model Analysis for measuring insulin resistance [16]. FIRI is the product of fasting insulin and fasting glucose levels. In order to facilitate interpretation, FIRI was scaled to a mean value of 1 by dividing by the sample mean value (63.3) [15].

\section{Exclusions}

Of the NAS cohort of 2,280 males, 10 were lost to follow-up, 389 had died and 422 had ceased to participate in regular physical examinations (although they continued to complete questionnaires) when insulin values were being measured. Of the remaining 1,459 subjects, 1,223 were examined during the relevant period and so were able to contribute insulin data. One of these subjects was excluded because of missing baseline spirometry and 40 were excluded because their baseline fasting and PC glucose levels were consistent with diabetes $(n=2)$ or impaired glucose tolerance $(n=38)$ according to standard criteria [17]. An additional 104 subjects with diabetes at follow-up (de- fined as either a physician diagnosis of diabetes or using antidiabetic medication) were excluded because treatment would influence their insulin levels. Finally, 27 subjects with one or more missing values from their baseline athropometry or PC glucose records were excluded, leaving 1,050 subjects with all data required for the analysis.

The 1,130 subjects with missing insulin values were significantly older (44.4 compared with $41.6 \mathrm{yrs}, \mathrm{p}=0.0001$ ) and more centrally obese (AC/HB 2.56 compared with 2.54 , $\mathrm{p}=0.016)$, and had lower FVC (4.76 compared with 4.95 $\mathrm{L}, \mathrm{p}=0.0001$ ) and FEV1 (3.77 compared with $3.94 \mathrm{~L}, \mathrm{p}=$ $0.0001)$ at baseline, by unpaired t-test, when compared with the 1,050 subjects with data available for analysis.

\section{Analysis}

SAS [18] (ver. 6.09) was used for all analyses. FVC, FEV1 and MMEF at study entry were adjusted before analysis because they are strongly influenced by age and stature [19]. Residuals (predicted values subtracted from observed values) from linear regression on age and height were estimated for each subject. These are referred to as adjusted FVC, adjusted FEV1 and adjusted MMEF.

The association between quintile of baseline ventilatory function and mean follow-up FIRI and fasting insulin levels was tested by analysis of variance (ANOVA). The skewed distributions of fasting insulin and FIRI were substantially improved by a logarithmic transformation, so log-transformed values were used for ANOVA.

Nonparametric criteria were used to define two indicators of relative insulin resistance. Subjects in the top quintile of the distribution of fasting insulin values were defined as being relatively insulin resistant. The same procedure was applied to FIRI values. The relative risk for each of these two outcomes was modelled using logistic regression. Separate models for each of the three adjusted baseline measures of ventilatory function were estimated, with control for potential confounders measured at baseline including age, life-time tobacco smoking (pack-years), relative obesity (BMI) and fat distribution pattern (AC/HB). Finally, since baseline insulin measures were not available, baseline PC glucose was added to each model to examine the effect of bias from glucose intolerance at the time of spirometry.

Table 1. - Characteristics of the subjects by age and height-adjusted forced vital capacity (FVC) quintile at study entry (Normative Aging Study, 1961-1991)

\begin{tabular}{|c|c|c|c|c|c|c|}
\hline \multirow[t]{2}{*}{ Characteristic } & \multicolumn{5}{|c|}{ Baseline adjusted FVC quintile ( $\mathrm{n}=210$ each) } & \multirow{2}{*}{$\begin{array}{l}\text { All combined } \\
\quad(n=1050)\end{array}$} \\
\hline & 1 (lowest) & 2 & 3 & 4 & 5 (highest) & \\
\hline Age yrs & $41.6 \pm 7.8$ & $41.1 \pm 7.3$ & $41.4 \pm 7.6$ & $41.1 \pm 8.1$ & $41.8 \pm 8.2$ & $41.4 \pm 7.8$ \\
\hline Body mass index $\mathrm{kg} \cdot \mathrm{m}^{-2}$ & $25.9 \pm 2.8$ & $25.8 \pm 2.8$ & $25.4 \pm 2.51$ & $25.5 \pm 2.5$ & $25.5 \pm 2.5$ & $25.6 \pm 2.5$ \\
\hline $\mathrm{AC} / \mathrm{HB}$ & $2.57 \pm 0.14$ & $2.54 \pm 0.16$ & $2.54 \pm 0.15$ & $2.50 \pm 0.14$ & $2.51 \pm 0.15$ & $2.53 \pm 0.15$ \\
\hline Tobacco use pack-yrs & $19.0 \pm 18.4$ & $17.1 \pm 16.8$ & $15.1 \pm 18.8$ & $14.1 \pm 18.0$ & $11.4 \pm 14.8$ & $15.3 \pm 17.6$ \\
\hline FVC L & $4.23 \pm 0.55$ & $4.70 \pm 0.46$ & $4.95 \pm 0.46$ & $5.24 \pm 0.48$ & $5.76 \pm 0.52$ & $4.97 \pm 0.72$ \\
\hline Adjusted FVC* & $-0.76 \pm 0.29$ & $-0.28 \pm 0.09$ & $-0.003 \pm 0.08$ & $0.29 \pm 0.09$ & $0.76 \pm 0.24$ & $0.0 \pm 0.55$ \\
\hline FEV1 L & $3.44 \pm 0.53$ & $3.76 \pm 0.46$ & $4.00 \pm 0.46$ & $4.17 \pm 0.46$ & $4.48 \pm 0.49$ & $3.96 \pm 0.60$ \\
\hline Adjusted $\mathrm{FEV}_{1} *$ & $-0.54 \pm 0.36$ & $-0.21 \pm 0.30$ & $0.01 \pm 0.30$ & $0.23 \pm 0.30$ & $0.50 \pm 0.35$ & $0.0 \pm 0.48$ \\
\hline MMEF $L \cdot \mathrm{s}^{-1}$ & $2.30 \pm 0.69$ & $2.44 \pm 0.76$ & $2.52 \pm 0.72$ & $2.61 \pm 0.75$ & $2.62 \pm 0.72$ & $2.50 \pm 0.74$ \\
\hline Adjusted MMEF* & $-0.20 \pm 0.67$ & $-0.07 \pm 0.73$ & $0.03 \pm 0.70$ & $0.12 \pm 0.73$ & $0.13 \pm 0.69$ & $0.0 \pm 0.71$ \\
\hline Fasting insulin pmol. $\mathrm{L}^{-1+}$ & $89.4 \pm 56.6$ & $87.0 \pm 55.8$ & $73.3 \pm 41.6$ & $74.0 \pm 47.5$ & $74.4 \pm 45.2$ & $79.60 \pm 50.1$ \\
\hline $\mathrm{FIRI}^{+}$ & $1.13 \pm 0.80$ & $1.11 \pm 0.80$ & $0.92 \pm 0.58$ & $0.91 \pm 0.63$ & $0.93 \pm 0.61$ & $1.00 \pm 0.70$ \\
\hline
\end{tabular}

Data are mean \pm SD. + : at follow-up examination. All other values are from study entry examination. *: residuals (observed-predicted) from linear regression on age and height. AC/HB: ratio of abdominal circumferences to hip breadth; FEV1: forced expiratory volume in one second; MMEF: maximal mid-expiratory flow; FIRI: fasting insulin resistance index. 


\section{Results}

The mean interval between study entry examination and insulin measurement was 20.9 yrs $(S D=2.6)$. Age, height, FVC, FEV1, MMEF, adjusted FVC, adjusted FEV1, adjusted MMEF, BMI, AC/HB and pack-years of smoking at study entry, together with fasting insulin and FIRI at follow-up, are shown in table 1, by adjusted FVC quintile and for all subjects combined. The mean values for FIRI and for fasting insulin were substantially elevated in the lowest two quintiles of baseline FVC, suggesting that lower levels of ventilatory function at baseline were associated with higher levels of insulin and insulin resistance at follow-up, although this trend did not appear to be linear with increasing FVC. There were significant differences by ANOVA between quintiles of baseline adjusted $\mathrm{FVC}$ for AC/HB $(\mathrm{p}<0.0001)$, pack-years $(\mathrm{p}<0.0001)$, fasting insulin $(\mathrm{p}=0.0014)$ and FIRI $(\mathrm{p}=0.0009)$, but not for age $(\mathrm{p}=0.64)$ or BMI $(\mathrm{p}=0.068)$. The patterns by quintile of adjusted FEV1 and MMEF were very similar (not shown). These results suggest that adjustment in any models may be needed for potential confounders including smoking, obesity and fat distribution pattern.

Given the pattern seen in table 1, subjects were defined as being relatively insulin resistant if they were at or above the cut-off point defining the top quintile of either FIRI or fasting insulin. The lower limit of the top quintile for fasting insulin was $114.8 \mathrm{pmol} \cdot \mathrm{L}^{-1}$ (mean value for subjects above this cut-off point was $162.1 \mathrm{pmol} \cdot \mathrm{L}^{-1}$, SD 55.6). Mean FIRI had been adjusted to be 1.0 for the whole sample and the lower limit of the top quintile was 1.37 (mean value for subjects in the top quintile 2.08, SD 0.80). Table 2 summarizes the results of logistic regression models for the development of relative insulin resistance as defined by these cut-off points compared with all other subjects who were below that cut-off point. The relative risks in table 2 indicate that subjects with higher baseline adjusted ventilatory function values were less likely to be relatively insulin resistant by either criterion at follow-up.

When baseline PC glucose was added to the models summarized in table 2 to test for confounding by pre-existing glucose intolerance, the relative risks on baseline FVC, FEV1 and MMEF changed very little, as shown in table 3, suggesting that this was not an important source of bias in the findings.

Table 2. - Summary of logistic regression models for relatively high insulin resistance at follow-up, showing adjusted relative risks on baseline ventilatory function (Normative Aging Study, 1961-1991)

\begin{tabular}{|c|c|c|c|c|c|c|}
\hline \multirow[t]{2}{*}{ Predictor $^{+}$} & \multicolumn{3}{|c|}{ Fasting insulin } & \multicolumn{3}{|c|}{ FIRI } \\
\hline & Risk* & $95 \% \mathrm{CI}$ & p-value & Risk* & $95 \% \mathrm{CI}$ & p-value \\
\hline FVC L & 0.61 & $0.45-0.83$ & 0.002 & 0.56 & $0.42-0.75$ & 0.0001 \\
\hline FEV1 L & 0.49 & $0.35-0.70$ & 0.0001 & 0.45 & $0.32-0.63$ & 0.0001 \\
\hline $\begin{array}{c}\mathrm{MMEF} \\
\mathrm{L} \cdot \mathrm{S}^{-1}\end{array}$ & 0.68 & $0.53-0.87$ & 0.002 & 0.65 & $0.51-0.83$ & 0.0007 \\
\hline
\end{tabular}

+: baseline value adjusted for age and height; *: relative risk (adjusted for age, body mass index, ratio of abdominal circumference to hip breadth and pack-yrs of smoking) for being in the top quintile (compared with the lower four quintiles combined) at follow-up. FIRI: fasting insulin resistance index; CI: confidence interval; FVC: forced vital capacity; FEV1: forced expiratory volume in one second; MMEF: maximal mid-expiratory flow.
Table 3. - Summary of logistic regression models for relatively high insulin resistance at follow-up, showing adjusted relative risks on baseline ventilatory function with additional adjustment for baseline postchallenge glucose (Normative Aging Study, 1961-1991)

\begin{tabular}{|c|c|c|c|c|c|c|}
\hline \multirow{2}{*}{ Predictor $^{+}$} & \multicolumn{3}{|c|}{ Fasting insulin } & \multicolumn{3}{|c|}{ FIRI } \\
\hline & Risk* & $95 \% \mathrm{CI}$ & $\overline{\text { p-value }}$ & Risk* & $95 \% \mathrm{CI}$ & p-value \\
\hline & 0.59 & $0.43-0.79$ & 0.0005 & 0.64 & $0.47-0.87$ & 0.005 \\
\hline FEV1 L & 0.51 & $0.36-0.73$ & 0.0002 & 0.47 & $0.33-0.66$ & 0.0001 \\
\hline $\begin{array}{l}\mathrm{MMEF} \\
\mathrm{L} \cdot \mathrm{s}^{-1}\end{array}$ & 0.69 & $0.54-0.88$ & 0.003 & 0.66 & $0.51-0.85$ & 0.001 \\
\hline
\end{tabular}

$+:$ baseline value adjusted for age and height; *: relative risk (adjusted for age, body mass index, ratio of abdominal circumference to hip breadth, post-challenge glucose levels and packyrs of smoking) for being in the top quintile (compared with the lower four quintiles combined) at follow-up. FIRI: fasting insulin resistance index; CI: confidence interval; FVC: forced vital capacity; FEV1: forced expiratory volume in one second; MMEF: maximal mid-expiratory flow.

\section{Discussion}

The risk of being relatively insulin resistant, as measured by either fasting insulin or FIRI level, was negatively associated with baseline ventilatory function in a large group of initially healthy males followed up for $>20$ yrs. Higher mean FIRI and fasting insulin levels at follow-up were found in subjects with lower baseline levels of FVC and this pattern was statistically significant by ANOVA (table 1). When subjects in the highest quintile of FIRI and fasting insulin were defined as having the outcome of interest (relatively high insulin resistance), the risk of developing insulin resistance over the follow-up period was higher for subjects with lower FVC, FEV1 or MMEF at baseline, after multivariable adjustment for baseline age, relative adiposity, a central pattern of fat distribution, PC glucose and smoking in the logistic regression analyses summarized in tables 2 and 3 .

Specialized methods for measuring insulin resistance are not practicable for large cohorts such as the one studied here [20]. Moderately high correlations $(r=-0.68)$ have been reported in euglycaemic subjects between fasting insulin and whole body glucose uptake measured using the euglycaemic hyperinsulinaemic clamp method [21]. The FIRI has a good theoretical basis [16], but there has been some controversy about its use [22, 23]. Although fasting insulin or FIRI is not an ideal indicator of insulin resistance, the authors are not aware of evidence that measurement error in these measures is associated with ventilatory function. If there is nondifferential measurement error in the use of insulin values as indicators of insulin resistance, these findings are likely to have been biased towards a finding of no association and the analysis may have underestimated the true effect.

A substantial proportion of the NAS cohort did not have their insulin levels measured during the short interval when this was part of the NAS data collection. These males were significantly older, had a more central pattern of fat distribution and had lower levels of baseline ventilatory function than the subjects who had their insulin levels measured. The direction and strength of biases introduced by their effective exclusion is unknown. Most of these subjects had died or dropped out before insulin measurement, in some cases, presumably owing to adverse cardiovascular 
outcomes. If adverse cardiovascular outcomes were associated with insulin resistance and low baseline ventilatory function, these exclusions may have resulted in a bias towards a finding of no association.

The protocol used for spirometry at study entry was developed before the now widely accepted recommendations by the American Thoracic Society were published [24]. Baseline FVC and FEV1 values were adjusted for age and height by regression within the sample, so any systematic bias compared with more modern measurement methods is unlikely to have influenced the findings.

No measures of physical activity were available at baseline, so no adjustment could be made for this potential confounder of the relationship between insulin resistance and ventilatory function. Insulin measurements at baseline were not available, so the possibility that baseline insulin resistance has biased the results cannot be excluded. However, subjects with higher levels of baseline insulin resistance would, presumably, be more likely to develop diabetes during follow-up and thus be excluded from the analysis. In addition, since frankly diabetic subjects at baseline were excluded, higher levels of PC glucose would be expected among the more insulin-resistant participants and adjustment for baseline PC glucose would be expected to decrease the impact of any bias. The relative risks shown in table 3 differ only slightly from those in table 1, suggesting that bias from baseline insulin resistance has not substantially influenced the findings.

Insulin resistance is positively associated with age [25], obesity [11] and a central pattern of body fat distribution [26]. Ventilatory function is also associated with age, obesity [19] and fat distribution [27-29]. Confounding from these factors is not likely to be an explanation for the present findings because the analyses were appropriately adjusted.

Assuming the findings are valid, the task of explaining why insulin resistance is more likely to develop among subjects with decreased ventilatory function remains. Some underlying common cause may be associated with both decreased ventilatory function and the subsequent development of insulin resistance. A number of lines of evidence may prove worthy of further research.

Ventilatory function is partially determined by respiratory muscle strength. Although hand-grip strength is, at best, a very indirect indicator of respiratory muscle strength, lower levels of hand-grip strength have been found to predict the development of higher levels of fasting insulin in the NAS [30], raising the possibility that the underlying defect common to both impaired ventilatory function and impaired grip strength lies in skeletal muscle. It has been shown that the ability of insulin to increase skeletal blood flow is impaired in obese, insulin-resistant subjects [31]. Since skeletal muscle is an important site for insulin-mediated glucose disposal, it is possible that impaired ventilatory function and decreased skeletal muscle strength are both related to underlying changes in skeletal muscle that precede the development of insulin resistance. Alternatively, some underlying metabolic change that eventually leads to insulin resistance may initially induce diminished muscle strength and diminished ventilatory function.

In conclusion, nondiabetic subjects with decreased ventilatory function were more likely to develop higher insulin levels and insulin resistance. They would also be more likely to develop a range of associated cardiovascular risk factors because insulin resistance is associated with the development of noninsulin-dependent diabetes mellitus, essential hypertension and dyslipidaemia [11] and increased insulin levels have been found to predict the risk of coronary heart disease in prospective data [32]. Thus, the association of increased risk of mortality from coronary heart disease in subjects with decreased baseline ventilatory function may be at least partially mediated by the development of insulin resistance and hyperinsulinaemia.

\section{References}

1. Friedman G, Klatsky A, Siegelaub M. Lung function and risk of myocardial infarction and sudden cardiac death. $N$ Engl J Med 1976; 294: 1071-1075.

2. Kannel W, Hubert H, Lew E. Vital capacity as a predictor of cardiovascular disease: the Framingham study. $\mathrm{Am}$ Heart J 1983; 105: 311-315.

3. Persson C, Bengtsson C, Lapidus L, Rybo E, Thringer G, Wedel H. Peak expiratory flow rate and risk of cardiovascular disease and death: a 12-year follow-up of participants in the population study of women in Gothenburg, Sweden. Am J Epidemiol 1986; 124: 942-948.

4. Lange P, Nyboe J, Jensen G, Schnohr P, Appleyard M. Ventilatory function impairment and risk of cardiovascular death and of fatal or non-fatal myocardial infarction. Eur Respir J 1991; 4: 1080-1087.

5. Beaty T, Cohen B, Newill C, Menkes H, Diamond E, Chen C. Impaired pulmonary function as a risk factor for mortality. Am J Epidemiol 1982; 116 (1): 102-113.

6. Marcus E, Buist S, Curb D, et al. Correlates of FEV1 and prevalence of pulmonary conditions in Japanese-American men. Thorax 1988; 138: 1398-1404.

7. Lange P, Groth S, Kastrup J, et al. Diabetes mellitus, plasma glucose and lung function in a cross-sectional population study. Eur Respir J 1989; 2: 14-19.

8. Burchfiel C, Curb D, Sharp D, et al. Distribution and correlates of insulin in elderly men: the Honolulu heart program. Arterioscl Thromb Vasc Biol 1995; 15: 2213-2221.

9. Lange P, Groth S, Mortensen J, et al. Diabetes mellitus and ventilatory capacity: a five year follow-up study. Eur Respir J 1990; 3: 288-292.

10. Hall J, Sumrners R, Brands M, Keen H, Alonso-Galicia $\mathrm{M}$. Resistance to the metabolic actions of insulin and its role in hypertension. Am J Hypertens 1994; 7: 772-788.

11. DeFronzo R, Ferrannini E. Insulin resistance: a multifaceted syndrome responsible for NIDDM, obesity, hypertension, dyslipidemia and atherosclerotic cardiovascular disease. Diabetes Care 1991;14: 173-194.

12. Bell B, Rose C, Damon H. The normative aging study: an interdisciplinary and longitudinal study of health and aging. Aging Hum Dev 1972; 3: 5-17.

13. Willett W. Anthropometric measures and body composition. In: MacMahon B, ed. Nutritional Epidemiology, 1st Edn. Monographs in Epidemiology and Biostatistics, Vol. 15, New York, Oxford University Press, 1990; pp. 217244.

14. Kory R, Callahan R, Boren H, Syner J. The Vererans Administration-Army Cooperative Study of Pulmonary Function: I. Clinical spirometry in normal men. Am J Med 1961; 30: 243-258.

15. Duncan MH, Singh BM, Wise PH, Carter G. A simple measure of insulin resistance. Lancet 1995; 346: 120-121.

16. Mathews DR, Hosker JP, Rudenski AS, Naylor BA, Treacher DF, Turner RC. Homeostasis model assessment: 
insulin resistance and $\beta$-cell function from fasting plasma glucose and insulin concentrations in man. Diabetologia 1985; 28: 412-419.

17. World Health Organization. Diabetes mellitus. Report of a WHO study group. Geneva, World Health Organization, 1985; Technical Report Series 727.

18. SAS Institute. Statistical analysis system, ver. 6.12. Cary, NC, SAS Institute, 1997.

19. Schoenberg J, Beck G, Bouhuys A. Growth and decay of pulmonary function in healthy blacks and whites. Respir Physiol 1978; 33: 367-393.

20. Anderson R, Hamman R, Savage P, et al. Exploration of simple insulin sensitivity measures derived from frequently sampled intravenous glucose tolerance (FSGIT) tests. Am J Epidemiol 1995; 142: 724-732.

21. Laakso M. How good a marker is insulin level for insulin resistance? Am J Epidemiol 1993; 137: 959-965.

22. Cleland SJ, Petrie JR, Morris AD, Ueda S, Dorrian CA, Connell JMC. FIRI: a fair insulin resistance index. Lancet 1996; 347: 770.

23. Del Prato S, Pozzilli P. FIRI: fasting or false insulin resistance index? Lancet 1996; 347: 132.

24. American Thoracic Society. Standardization of spirometry. Am Rev Respir Dis 1979; 119: 831-838.

25. Coleman E, Toth M, Katzel L, Fonong T, Gardner A, Poehlman E. Body fatness and waist circumference are independent predictors of the age-associated increase in fasting insulin levels in healthy men and women. Int $J$ Obesity 1995; 19: 798-803.

26. Bjorntorp P. The regulation of adipose tissue distribution in humans. Int J Obesity 1996; 20: 291-302.

27. Collins L, Hoberty P, Walker J, Fletcher E, Peiris A. The effect of body fat distribution on pulmonary function tests. Chest 1995; 107: 1298-1302.

28. Lazarus R, Sparrow D, Weiss ST. Effects of obesity and fat distribution on ventilatory function. Chest 1997; 111: 891-898.

29. Lazarus R, Gore C, Booth M, Owen N. Effects of body composition and fat distribution on ventilatory function in adults. Am J Clin Nutr 1998; 68: 35-41.

30. Lazarus R, Sparrow D, Weiss ST. Hand grip strength and insulin levels: cross sectional and longitudinal associations in the Normative Aging Study. Metabolism 1997; 46: 1266-1269.

31. Laakso M, Edelman SV, Brechtel G, Baron AD. Decreased effect of insulin to simulate skeletal blood flow in obese man: a novel mechanism for insulin resistance. $J$ Clin Invest 1990; 85: 1844-1852.

32. Perry I, Wannarnethee SG, Whincup P, Shaper AG, Walker M, Alberti KG. Serum insulin and incident coronary heart disease in middle-aged British men. Am J Epidemiol 1996; 144: 224-234. 